\title{
Adiabatic Plasma Rotations in Orthogonal Coordinate Systems
}

\author{
Ricardo L. Viana \\ Departamento de Física, Universidade Federal do Paraná, \\ Caixa Postal 19081, 81531-990, Curitiba, Paraná, Brazil
}

Received on 26 July, 2000

\begin{abstract}
An equation for MHD stationary equilibrium of rotating plasmas in the azimuthal direction is derived in the case of an orthogonal curvilinear coordinate system. The basic assumptions we made are: (i) there is an ignorable coordinate so that surface quantities are independent of it; (ii) the entropy is a surface quantity.
\end{abstract}

\section{Introduction}

Azimuthal rotation in Tokamaks and other fusion machines is observed, for example, when the confined plasma is subjected to neutral beam heating. The impacts of the beam particles with plasma electrons and ions amounts to a net momentum transfer with causes rotation in the toroidal direction $[1,2]$. Plasma rotation with high Mach numbers have been observed in almost all operating regimes of Tokamaks [3, 4], as well as in reversed-field pinches [5].

A key problem in the theoretical study of azimuthal rotation is whether such a plasma flow could coexist with a state of MHD (stationary) equilibrium. The answer turns to be positive provided some requirements are fulfilled by the system. Without resistivity, Alfvén's theorem says that magnetic field lines rotate rigidly with the plasma. If axissymetry exists, field lines lie on magnetic flux surfaces with topology of tori and characterized by surface quantities, like the transversal magnetic flux. The set of ideal MHD equations allows us to derive a partial differential equation for it $[6,7]$. Maschke and Perrin $[8,9]$ obtained a MHD equilibrium equation for azimuthal plasma flows supposing that either the temperature or the entropy were surface quantities. They have considered only cylindrical coordinates, having obtained exact analytical solutions for the transversal magnetic flux. There are a few other solved cases in cylindrical [10] and spherical [11] geometries, but considering the temperature as a surface quantity.

The case where the plasma flow is adiabatic, however, demands the use of the entropy as a surface quantity. This is particularly important in the case of anisotropic plasmas, where a double-adiabatic theory is necessary to describe the situation $[12,13]$. To apply the MHD equilibrium theory for realistic magnetic confinement schemes, one would need an equilibrium equation in a general curvilinear coordinate system. In a previous paper [14] an equilibrium equation for plasmas with azimuthal rotation was derived, assuming that the temperature was a surface quantity, in an orthogonal curvilinear coordinate system. In this paper we will derive a similar equation, but with the plasma entropy as a surface quantity, according the methodology introduced by Maschke and Perrin [8].

This paper is organized as follows: in the second section we outline the basic equations and thermodynamical relations to be used, the magnetic field and velocity representations. In section III we use these equations to obtain a pressure equilibrium equation, which is supplemented by a Bernoulli-like algebraic equation. Section IV presents a particular form of this equation, obtained by a special choice of some surface quantities. Section V discusses how the, general equations look like in some coordinate systems, like cylindrical, spherical and prolate spheroidal ones.

\section{Basic Equations}

In a stationary MHD equilibrium theory, we consider an ideal (infinite conductivity) plasma of electrons and singly charged ions, where all partial time derivatives vanish, but allowing a constant velocity. The corresponding set of MHD equations are, in S.I. units [15]:

$$
\begin{array}{r}
\nabla \cdot(\rho \mathbf{v})=0, \\
\rho(\mathbf{v} . \nabla) \mathbf{v}=-\nabla p+\mathbf{j} \times \mathbf{B},
\end{array}
$$




$$
\begin{array}{r}
\nabla . \mathbf{B}=0, \\
\nabla \times \mathbf{B}=\mu_{0} \mathbf{j}, \\
\nabla \times \mathbf{E}=0, \\
\mathbf{E}+\mathbf{v} \times \mathbf{B}=0,
\end{array}
$$

where $\rho=n\left(m_{e}+m_{\imath}\right)$ is the mass density, $n$ is the particle number density and $m_{e}, m_{\imath}$ are the electronic and ionic masses, respectively. $\mathbf{v}, p, \mathbf{E}, \mathbf{B}$, and $\mathbf{j}$ are the velocity, pressure, electric field, magnetic field and plasma current density, respectively.

The specific internal energy $e$ and the specific entropy $S$ satisfy Gibbs' equation

$$
d e=T d S+\frac{p}{\rho^{2}} d \rho
$$

and the specific enthalpy $h$ satisfies the thermodynamical identity

$$
d p=\rho(d h-T d S)
$$

We also suppose the plasma as an ideal gas, obeying the equation

$$
p=\rho \bar{R} T=n k T,
$$

where $T$ is the plasma temperature (sum of electronic and ionic temperatures), $\bar{R}$ and $k$ being the gas constant and the Boltzmann constant, respectively.

We assume that the thermodynamical processes involved with plasma rotation are adiabatic, so that the caloric equation of state holds

$$
p=A(S) \rho^{\gamma}
$$

where $A=A(S)$ is a constant depending only on the entropy, and $\gamma=5 / 3$ is the ratio of specific heats. The internal energy in this case is

$$
e=A(S) \frac{\rho^{\gamma-1}}{\gamma-1}=\frac{h}{\gamma},
$$

so that the plasma temperature is given by

$$
T=\left.\frac{\partial e}{\partial S}\right|_{\rho}=\frac{\rho^{\gamma-1}}{\gamma-1} \frac{d A}{d S} .
$$

The adiabatic sound velocity in the plasma is given by Eqs. (8) and (11) as

$$
c_{s}=\sqrt{\left.\frac{\partial p}{\partial \rho}\right|_{S}}=\sqrt{\gamma \bar{R} T} .
$$

For an adiabatic process we have an entropy constant in time, and so is any function of entropy:

$$
\frac{d A(S)}{d t}=\frac{d}{d t}\left(\frac{p}{\rho^{\gamma}}\right)=0
$$

However, the convective derivative of $A$ is given by

$$
\frac{d A(S)}{d t}=\frac{\partial A}{\partial t}+\mathbf{v} \cdot \nabla A=0
$$

where the partial derivative vanishes for equilibria, giving the following relation for $S$

$$
\mathbf{v} \cdot \nabla S=0 .
$$

We will denote by $\left(x^{1}, x^{2}, x^{3}\right)$ the contravariant coordinates in a curvilinear coordinate system, and assume that $x^{3}$ is an ignorable coordinate, such that surface quantities do not depend on it. $\hat{e}_{\imath}(\imath=1,2,3)$ denote the covariant basis vectors for this system, and $g_{\imath \jmath}=\hat{e}_{\imath} \cdot \hat{e}_{\jmath}$ are the covariant components of the metric tensor ${ }^{1}$. Only orthogonal coordinate systems, in which $g_{\imath \jmath}=0$ for $\imath \neq \jmath$, will be considered here.

The representation of a solenoidal magnetic field in terms of two scalar surface functions is

$$
\mathbf{B}\left(x^{1}, x^{2}\right)=\frac{\hat{e}_{3}}{g_{33}} \times \nabla \Psi\left(x^{1}, x^{2}\right)-\mu_{0} I\left(x^{1}, x^{2}\right) \frac{\hat{e}_{3}}{g_{33}},
$$

where $\Psi$ and $I$ are the transversal flux and current functions, respectively. A plasma flow satisfying mass conservation Eq. (1) may be a rotation with constant angular frequency $\Omega$ along the $\hat{e}_{3}$ (azimuthal) direction

$$
\mathbf{v}\left(x^{1}, x^{2}\right)=\Omega\left(x^{1}, x^{2}\right) \hat{e}_{3}
$$

which satisfies Ferraro's iso-rotation law

$$
\nabla \Omega \times \nabla \Psi=0
$$

so that $\Omega=\Omega(\Psi)$ is also a surface quantity.

\section{The equation of motion}

The equation of motion for the rotating plasma in this ideal MHD theory is derived from the momentum balance equation (2), in which we have used the magnetic field and velocity representations, and Ampére's law, Eq. (4). A standard calculation would give the plasma current density in terms of the surface functions $\Psi$ and $I$

\footnotetext{
${ }^{1}$ See the appendix of Ref. [14] for further details about curvilinear coordinate systems
} 


$$
\mathbf{j}\left(x^{1}, x^{2}\right)=\frac{1}{\mu_{0}} \Delta^{*} \Psi\left(\frac{\hat{e}_{3}}{g_{33}}\right)-\frac{1}{\sqrt{g}}\left(\frac{\partial I}{\partial x^{2}} \hat{e}_{1}-\frac{\partial I}{\partial x^{1}} \hat{e}_{2}\right),
$$

where the generalized Shafranov operator is given by

$$
\Delta^{*} \Psi=g_{33} \nabla \cdot\left(\frac{\nabla \Psi}{g_{33}}\right)=\nabla^{2} \Psi+g_{33} \nabla \Psi \cdot \nabla\left(\frac{1}{g_{33}}\right) .
$$

The velocity-dependent term in the momentum balance equation Eq. (2) may be written as

$$
\rho(\mathbf{v} . \nabla) \mathbf{v}=-\frac{1}{2} \rho \Omega^{2} \nabla g_{33},
$$

A straightforward algebra leads to

$$
\left(\Delta^{*} \Psi+\frac{\mu_{0}^{2}}{2} \frac{d I^{2}}{d \Psi}\right) \nabla \Psi=-\mu_{0} g_{33}\left(\nabla p-\frac{\rho \Omega^{2}}{2} \nabla g_{33}\right) .
$$

Using the representation (18) for velocity, the condition (16) is identically satisfied due to axisymmetry, so it does not give any further information about the plasma entropy. Hence, we will assume that the entropy is a surface quantity: $S=S(\Psi)$. Using (7) we have for the pressure gradient $\nabla p=\rho(\nabla h-T \nabla S)$. Using also the isorotation law (19) we obtain

$$
\left[\Delta^{*} \Psi+\frac{\mu_{0}^{2}}{2} \frac{d I^{2}}{d \Psi}-\mu_{0} g_{33} \rho\left(T \frac{d S}{d \Psi}-g_{33} \Omega \frac{d \Omega}{d \Psi}\right)\right] \nabla \Psi=-\mu_{0} g_{33} \rho \nabla\left(h-\frac{1}{2} \Omega^{2} g_{33}\right) .
$$

Let us define

$$
\Theta=h-\frac{1}{2} \Omega^{2} g_{33},
$$

as a kind of centrifugally corrected enthalpy. Making the cross product with $\nabla \Psi$ it follows that $\Theta$ is also a surface quantity. In this way, for arbitrary and non-vanishing $\nabla \Psi$ we obtain the form of Maschke-Perrin equation with entropy as a surface function

$$
\Delta^{*} \Psi+\frac{\mu_{0}^{2}}{2} \frac{d I^{2}}{d \Psi}+\mu_{0} g_{33} \rho\left[g_{33} \Omega \frac{d \Omega}{d \Psi}+\frac{d \Theta}{d \Psi}-T \frac{d S}{d \Psi}\right]=0 .
$$

In the limit of vanishing rotation $\Omega=0$ we have simply $\Theta=h$, and using Gibbs' equation (7) we re-obtain the Grad-Shafranov equation for static equilibria

$$
\Delta^{*} \Psi+\frac{\mu_{0}^{2}}{2} \frac{d I^{2}}{d \Psi}+\mu_{0} g_{33} \frac{d p}{d \Psi}=0 .
$$

From Eq. (12), and since $S$ and $A$ are surface quantities, we have for the temperature-dependent term in (26)

$$
T \frac{d S}{d \Psi}=\frac{\rho^{\gamma-1}}{\gamma-1} \frac{d A}{d \Psi} .
$$

By the same token, then density can be eliminated by noting that, from (11), the enthalpy is

$$
h=\frac{\gamma}{\gamma-1} A(S) \rho^{\gamma-1},
$$

which gives

$$
\rho=\left[\frac{\gamma-1}{\gamma A}\left(\Theta+\frac{1}{2} \Omega^{2} g_{33}\right)\right]^{\frac{1}{\gamma-1}},
$$

showing that, in this model, the plasma density is not a surface function neither an independent variable, rather being determined by the knowledge of the surface functions $A, \Theta$ and $\Omega$. These are fixed, on the other hand, by the solution of the equilibrium equation itself, since it contains four arbitrary surface functions (the fourth being the current function $I$ ). The complete system of equations contains Eq. (26) and an algebraic relation defining $\Theta$, Eq. (25). With appropriate boundary conditions it becomes a well-posed model.

\section{Alternative form of the equi- librium equation}

Let us define, for later convenience

$$
\eta=\frac{\gamma}{\gamma-1}
$$


and rewrite the equilibrium equation (26) in the form

$$
\Delta^{*} \Psi+\frac{\mu_{0}^{2}}{2} \frac{d I^{2}}{d \Psi}+\mu_{0} g_{33}\left(1+\frac{g_{33} \Omega^{2}}{2 \Theta}\right)^{\eta-1} \Pi=0
$$

where

$$
\Pi \equiv\left(\frac{\Theta}{\eta A}\right)^{\eta-1}\left\{g_{33}\left[\Omega \Omega^{\prime}-\left(\frac{\eta-1}{\eta}\right) \frac{A^{\prime}}{A} \frac{\Omega^{2}}{2}\right]+\Theta^{\prime}-\left(\frac{\eta-1}{\eta}\right) \frac{A^{\prime}}{A} \Theta\right\},
$$

and the primes denote differentiation with respect to the magnetic flux. It may be rewritten as

$$
\Pi=\left[\left(\frac{\Theta}{\eta}\right)^{\eta} A^{1-\eta}\right]^{\prime}+g_{33}\left[\left\{\left(\frac{\Theta}{\eta}\right)^{\eta}\right\}^{\prime} A^{1-\eta} \frac{\Omega \Omega^{\prime}}{\Theta^{\prime}}+\left(\frac{\Theta}{\eta}\right)^{\eta}\left(A^{1-\eta}\right)^{\prime} \frac{\Omega^{2}}{2 \Theta}\right] .
$$

Thus the equilibrium equation assumes the following form

$$
\begin{array}{r}
\Delta^{*} \Psi+\frac{\mu_{0}^{2}}{2} \frac{d I^{2}}{d \Psi}+\mu_{0} g_{33}\left(1+\frac{g_{33} \Omega^{2}}{2 \Theta}\right)^{\eta-1} \times \\
\left\{\left[\left(\frac{\Theta}{\eta}\right)^{\eta} A^{1-\eta}\right]^{\prime}+g_{33}\left[\left\{\left(\frac{\Theta}{\eta}\right)^{\eta}\right\}^{\prime} A^{1-\eta} \frac{\Omega \Omega^{\prime}}{\Theta^{\prime}}+\left(\frac{\Theta}{\eta}\right)^{\eta}\left(A^{1-\eta}\right)^{\prime} \frac{\Omega^{2}}{2 \Theta}\right]\right\}=0
\end{array}
$$

which has four surface functions to be specified: $I(\Psi)$, $A(\Psi), \Omega(\Psi)$, and $\Theta(\Psi)$. According to Ref. [8] we choose the latter two such that

$$
\frac{\Omega^{2}(\Psi)}{\Theta(\Psi)}=\frac{\omega^{2}}{\ell^{2}}
$$

where $\omega$ is a constant, and $\ell$ is a characteristic length of the system.

It is also convenient to introduce an auxiliary surface function

$$
G(\Psi)=\left(\frac{\Theta}{\eta}\right)^{\eta} A^{1-\eta}
$$

The physical meaning of this function comes from the static limit of the problem. From (25) we have $\Theta=h$, and using (10), (11), and (31), a simple calculation shows that $G \rightarrow p$, so we may regard $G$ as a kind of centrifugally corrected plasma pressure.

With help of (36) and (37) we rewrite once more the equilibrium equation in a very concise form as

$$
\Delta^{*} \Psi+\frac{\mu_{0}^{2}}{2} \frac{d I^{2}}{d \Psi}+\mu_{0} g_{33}\left(1+\frac{g_{33}}{2} \frac{\omega^{2}}{\ell^{2}}\right)^{\eta} \frac{d G}{d \Psi}=0
$$

where the number of surface functions to be specified has been reduced to just two: $G$ and $I$.

The Mach number for the plasma azimuthal notation is given by

$$
\mathcal{M}=\frac{\left|v_{<3>}\right|}{c_{s}}=\frac{\sqrt{g_{33}} \Omega}{\sqrt{\gamma \bar{R} T}},
$$

where $v_{<3>}$ is the "physical" component of the velocity in the azimuthal direction, and we have used Eq. (13). It is worth-noting that the positive-definiteness of the function $\Theta$ (since $\Theta=(\Omega \ell / \omega)^{2}>0$ ) imposes a limit on the possible values of the Mach number for our hypothesis (36) to be valid. From (25) it follows that $h>\left(\Omega^{2} g_{33} / 2\right)$. Now, using (11) and (13) there results that $c_{s}^{2}=h(\gamma-1)$. Combining the two preceding inequalities we have the following condition for the rotational Mach number

$$
\mathcal{M}^{2}<\frac{2}{\gamma-1}
$$

For $\gamma=5 / 3$, we have that $\mathcal{M}<\sqrt{3}$, i.e., the plasma rotational velocity should not be larger than $\approx 1.73$ times the adiabatic sound velocity. This is a rather restrictive condition since it precludes most of the supersonic regime.

Finally it follows from the above analysis that the constant $\omega$ which have entered in the equilibrium equation is related to the Mach number by the following formula

$$
\omega^{2}=\frac{\ell^{2}}{g_{33}}\left[\frac{(\gamma-1) \mathcal{M}^{2}}{1-(\gamma-1) \mathcal{M}^{2} / 2}\right] .
$$

For vanishing rotation we have simply $\omega^{2}=\ell^{2} / g_{33}$, i.e., it is just a geometrical factor. 


\section{Particular cases}

Cylindrical geometry. In this case we have $\left(x^{1}, x^{2}, x^{3}\right)=(R, Z, \phi)$, and take the angle $\phi$ as our ignorable coordinate, so that $g_{33}=R^{2}$. The characteristic length in this case will be written $\ell=R_{0}$, which may represent the radius of the cylindrical conductor shell surrounding the rotating plasma, with $\Psi\left(R_{0}, \phi, Z\right)=0$ as a convenient boundary condition. The Shafranov operator (21) in this case is

$$
\Delta^{*} \Psi=R \frac{\partial}{\partial R}\left(\frac{1}{R} \frac{\partial \Psi}{\partial R}\right)+\frac{\partial^{2} \Psi}{\partial Z^{2}},
$$

and the equilibrium equation (38) is

$$
\Delta^{*} \Psi+\frac{\mu_{0}^{2}}{2} \frac{d I^{2}}{d \Psi}+\mu_{0} R^{2}\left(1+\frac{\omega^{2}}{2} \frac{R^{2}}{R_{0}^{2}}\right)^{\eta} \frac{d G}{d \Psi}=0
$$

In units where $\mu_{0}=1$, and after the following changes of notation: $\Delta^{*} \rightarrow \mathcal{L}, \Psi \rightarrow F, \omega \rightarrow \Omega$, and $G \rightarrow p_{s}$, this equation reduces to Eq. (3.8) of Ref. [8]. This partial differential equation has to be supplemented by an algebraic equation defining $\Theta$, obtained from (25) as

$$
\Theta=h-\frac{1}{2} R^{2} \Omega^{2}
$$

Since $\Omega$ is the plasma angular velocity, the term $R^{2} \Omega^{2} / 2$ is the specific rotational kinetic energy. On a given flux surface one has $\Psi=$ constant, and so any surface function like $\Theta(\Psi)$. As in fluid mechanics, we may write down a Bernoulli-type equation of the form $h-\left(R^{2} \Omega^{2} / 2\right)=$ const. along the points of a stream line of the plasma flow $(\mathbf{v} \times \mathbf{d l}=\mathbf{0})$. This is possible because the entropy is a surface quantity $(\mathbf{v} \cdot \nabla S=0)$, being an absent feature in isothermal plasma flows (in which the temperature is a surface quantity).

The equilibrium equation contains two surface functions whose profiles have to be specified a priori. In Ref.
[8] linear profiles were chosen for both

$$
\begin{array}{r}
G(\Psi)=\frac{\varpi}{R_{0}^{4}}\left(\Psi-\Psi_{0}\right), \\
I^{2}(\Psi)=I_{0}^{2}+2 \frac{M}{R_{0}^{2}}\left(\Psi-\Psi_{0}\right),
\end{array}
$$

with $\varpi, \Psi_{0}, I_{0}$ and $M$ are model constants. Maschke and Perrin [8] have found an exact and analytical solution for the equilibrium equation (43), using elementary functions only. Some features present in their solution may be cited here. First, the magnetic axis (defined as the extremum of the magnetic flux $\Psi$ at the $z=0$ plane) is displaced outwards due to the centrifugal effect. The magnitude of the displacement is proportional to $\Omega$. Also, the magnetic flux surfaces and pressure (isobaric) surfaces do not coincide, as it occurs in the static case. This is easily understood by noting that the pressure $p$ is no longer a surface quantity. In a later paper [9] an approximate analytical solution was given to a combined poloidal and toroidal adiabatic rotation.

Up to our knowledge, there are no further analytical solutions of the equilibrium equation in cylindrical coordinates, when the entropy is a surface quantity. Even in the other case (with the temperature as a surface quantity) there are very few solutions.

Spherical geometry. This symmetry turns to be necessary to deal with some fusion plasma schemes, like field-reversed configurations; and astrophysical problems, like magnetic stars. The corresponding equation in spherical coordinates $\left(x^{1}, x^{2}, x^{3}\right)=(r, \theta, \varphi)$ follows from the general equation (38) by choosing $\varphi$ as an ignorable coordinate so that $g_{33}=r^{2} \sin ^{2} \theta$. Introducing a characteristic length $r_{0}$ we have the following equilibrium equation

$$
\frac{\partial^{2} \Psi}{\partial r^{2}}+\frac{1}{r^{2}}\left(\frac{\partial^{2} \Psi}{\partial \theta^{2}}-\cot \theta \frac{\partial \Psi}{\partial \theta}\right)+\frac{\mu_{0}^{2}}{2} \frac{d I^{2}}{d \Psi}+\mu_{0} r^{2} \sin ^{2} \theta\left(1+\frac{\omega^{2}}{2} \frac{r^{2}}{r_{0}^{2}} \sin ^{2} \theta\right)^{\eta} \frac{d G}{d \Psi}=0
$$

and the centrifugally corrected enthalpy is

$$
\Theta=h-\frac{1}{2} \Omega^{2} r^{2} \sin ^{2} \theta .
$$

Adopting the same linear profiles, Eqs. (45) and (46), used in the cylindrical case, and using the adimensional radius $x=r / r_{0}$ we have the following inhomogeneous partial differential equation

$$
\mathcal{L}^{*} \Psi+\mu_{0}^{2} \Psi+\mu_{0} \varpi x^{2} \sin ^{2} \theta\left(1-\frac{1}{2} \omega^{2} x^{2} \sin ^{2} \theta\right)^{\eta}=0
$$


where $\mathcal{L}^{*}=r_{0}^{2} \Delta^{*}$ is a reduced Shafranov operator in spherical coordinates. This equation does not seem to allow for an exact analytical solution for arbitrary $\eta$. Approximate solutions (for low $\omega$ ) may be investigated by using a binomial expansion of the $\eta$-dependent term. Retaining only the lowest order term the resulting equation would be similar to that solved in Ref. [11], with the difference that here the entropy is supposed to be a surface quantity, rather than the temperature. With similar boundary conditions (at a spherical conductor shell) the analytical solutions would be similar as well.

Prolate spheroidal geometry. This is a convenient coordinate systems to study MHD equilibria in spheromak-type and compact tori configurations. The $z$-axis is the symmetry axis and the curvilinear coordinates are $(\xi, \iota, \varphi)$, defined as [16]:

$$
x=r \cos \varphi, \quad y=r \sin \varphi, \quad z=c \cosh \xi \cos \iota,
$$

where $0 \leq \xi<\infty, 0 \leq \iota \leq \pi, 0 \leq \varphi<2 \pi$, and $r=c \sinh \xi \sin \iota, 2 c>0$ being the distance between the foci of coordinate surfaces, which turn to be prolate spheroids of semi-major axis $c \cosh \xi_{0}$ and semi-minor axis $c \sinh \xi_{0}$. For this system $g_{33}=c^{2} \sinh ^{2} \xi \sin ^{2} \iota$. Without loss of generality we may take $\ell=c$ as our characteristic length.

The generalized Shafranov operator in this coordinate system is

$$
\Delta^{*} \Psi=\frac{1}{c^{2}\left(\cosh ^{2} \xi-\cos ^{2} \iota\right)}\left[\frac{\partial^{2} \Psi}{\partial \xi^{2}}+\frac{\partial^{2} \Psi}{\partial \iota^{2}}-\operatorname{coth} \xi \frac{\partial \Psi}{\partial \xi}-\cot \iota \frac{\partial \Psi}{\partial \iota}\right],
$$

entering the equilibrium equation, which reads in this case

$$
\Delta^{*} \Psi+\frac{\mu_{0}^{2}}{2} \frac{d I^{2}}{d \Psi}+\mu_{0} c^{2} \sinh ^{2} \xi \sin ^{2} \iota\left(1+\frac{\omega^{2}}{2} \sinh ^{2} \xi \sin ^{2} \iota\right)^{\eta} \frac{d G}{d \Psi}=0 .
$$

In the static case, evidently there is no difference between taking the entropy or temperature as surface quantities, since these thermodynamical hypotheses are no longer necessary in this situation. This limit was studied in the early eighties by Kaneko and Takimoto [17], who have used profiles for $G \rightarrow p$ and $I^{2}$, linear and quadratic in $\Psi$, respectively. They also have found analytical solutions involving combinations of angular and radial spheroidal wave functions. Unlike the similar equation we have derived for spherical coordinates, it is very difficult to find a situation in which the Eq. (52) is amenable to analytic, even approximate, treatment.

\section{Conclusions}

We have extended the previous results regarding MHD equilibria with constant angular velocity in the azimuthal direction (using the entropy as a surface quantity) to a general context in which it suffices to specify three things about the coordinate system to be used: (i) an ignorable coordinate (with respect to it is the direction of the plasma flow); (ii) a corresponding component of the covariant metric tensor; (iii) a characteristic length, related to some obvious boundary condition or the plasma boundary itself.

The equilibrium equation in this case is a nonlinear elliptic partial differential equation where the main variable is the transversal magnetic flux function $\Psi$. Four surface quantities (which depend only on $\Psi$ ) have to be previously set up in order to reduce the number of dependent variables. This equation has to be supplemented by a Bernoulli-type algebraic equation which describes the centrifugally corrected plasma enthalpy.

A particular form of the equation is obtained by choosing a given form for two of the surface functions, reducing their number to just two. This choice, however, limits our treatment to rotations with Mach numbers up to $\sqrt{3}$. The plasma density is not a surface function, being determined in terms of the specified profiles chosen.

Besides the cylindrical case, which was already known in the literature, we have applied our general equation to the spherical and prolate spheroidal geometries, which are relevant to describe some magnetic confinement schemes like Spheromaks and compact tori. The equation in spherical coordinates is most likely sol- 
uble, at least in an approximate situation (low Mach number). The prolate spheroidal case seems to be only amenable to numerical treatment.

\section{Acknowledgments}

The author would like to acknowledge Dr. R. A. Clemente for useful discussions and valuable suggestions.

\section{References}

[1] M.G. Bell, Nuclear Fusion, 19, 33 (1979).

[2] S. Suckewer, H.P. Eubank, R.J. Goldston, E. Hinnov, and N.R. Sauthoff, Physical Review Letters, 43, 207 (1979).

[3] K. Brau, M. Bitter, R. J. Goldston, E. Hinnov, and N. R. Sauthoff, Physical Review Letters 26, 1643 (1979).

[4] R. C. Isler, et al. Nuclear Fusion 23, 1017 (1983).

[5] L. Carraro, M.E. Puiatti, F. Sattin, P. Scarin, and M. Valisa, Plasma Physics and Controlled Fusion 40, 1021 (1998).

[6] H.P. Zehrfeld, and B.J. Green, Nuclear Fusion, 10, 251 (1970).
[7] A.I. Morosov, L.S. and Solov'ev, (1980) in Reviews of Plasma Physics, Ed.: M. A. Leontovich, Vol. 8, Chap. 2 (Consultants Bureau, New York).

[8] E.K. Maschke, and H. Perrin, Plasma Physics, 22, 579 (1980).

[9] E.K. Maschke, and H. Perrin, Phys. Lett. A, 102, 106 (1984).

[10] R.A. Clemente, and R. Farengo, Physics of Fluids, 27, 776 (1984).

[11] R.L. Viana, R.A. Clemente, and S.R. Lopes, Plasma Physics and Controlled Fusion, 39, 197 (1997).

[12] R.A. Clemente, and R.L. Viana, Plasma Physics and Controlled Fusion, 41, 567 (1999).

[13] R.A. Clemente, and R.L. Viana, Brazilian Journal of Physics, 29, 457 (1999).

[14] R.L. Viana, International Journal of Theoretical Physics, 37, 2657 (1998).

[15] W.M. Stacey Jr., (1981). Fusion Plasma Analysis, Wiley, New York.

[16] P.M. Morse, and H. Feshbach, (1953). Methods of Theoretical Physics, McGraw Hill, New York, vol. 2, p. 1284 .

[17] S. Kaneko, and A. Takimoto, (1982). Proceedings of the Fourth US-Japan Workshop on Compact Toroids, Nagoya, Japan, p. 78. 